\title{
INTEGRATION OF FUNCTIONS WITH VALUES IN LOCALLY CONVEX SUSLIN SPACES
}

\author{
BY \\ G. ERIK F. THOMAS( $\left.{ }^{1}\right)$
}

\begin{abstract}
The main purpose of the paper is to give some easily applicable criteria for summability of vector valued functions with respect to scalar measures. One of these is the following: If $E$ is a quasi-complete locally convex Suslin space (e.g. a separable Banach or Fréchet space), $H \subset E^{\prime}$ is any total subset, and $f$ is an $E$-valued function which is Pettis summable relative to the ultra weak topology $\sigma(E, H)$. $f$ is actually Pettis summable for the given topology. (Thus any $E$-valued function for which the integrals over measurable subsets can be reasonably defined as elements of $E$ is Pettis summable.) A class of "totally summable" functions, generalising the Bochner integrable functions, is introduced. For these Fubini's theorem, in the case of a product measure, and the differentiation theorem, in the case of Lebesgue measure, are valid. It is shown that weakly summable functions with values in the spaces $D, E, S, D^{\prime}, E^{\prime}, S^{\prime}$, and other conuclear spaces, are ipso facto totally summable.
\end{abstract}

Introduction. The theory of Lebesgue integral on a general measure space has been extended to functions with values in a Banach space by Birkhoff [1], Bochner [2], Pettis [11] and others [10]. The extension of Pettis' definition of the integral to general locally convex spaces, required by present day analysis, offers no difficulty; nor do the proofs of the basic properties and of diverse convergence theorems present any new problems: it suffices to regard a locally convex space as a subspace of a product of Banach spaces.

At present the theory seems, however, to lack easily applicable criteria enabling one to decide whether a given vector valued function is summable. From what follows in this article it may be concluded that this deficiency is due in part to the absence, in the case of nonmetrisable locally convex spaces, of an adequate definition of measurability. Even in the case of Banach spaces it is known that without strong measurability assumptions the integral may present pathologies that make it less suitable for applications in analysis.

In the case of locally convex Suslin spaces the concept of "weak measurability" implies very strong coherence properties (Theorem 1 ( $(2)$ and Lemma B (§3)) and is sufficient for all purposes. Furthermore, locally convex

Received by the editors August 27, 1973 and, in revised form, March 8, 1974. AMS (MOS) subject classifications (1970). Primary 28A45, 46G10.

(1) Supported in part by NSF grant GP-30671. 
Suslin spaces, whose properties have recently been clarified by L. Schwartz [13] are very abundant. It seems that practically all separable spaces encountered in analysis are Suslin spaces. It seemed worthwhile therefore, to restrict attention to these spaces for the present, and to point out a number of properties which, while not concerned with the most general situations, may prove to be useful in some problems of analysis.

Contents. $\S 1$ contains a list of basic properties of summable functions (in the sense of Pettis) with values in locally convex spaces. This part of the article should be considered expository.

$\$ 2$ gives additional properties of the integral which are valid if the space is a Suslin space. Basic properties and examples of Suslin spaces are recalled at the beginning of this section.

$\$ 3$ contains integrability criteria valid in the case of locally convex Suslin spaces (Theorems 2-5).

$\S 4$ examines the particular case of nuclear spaces and their duals (Theorems $6,7)$.

$\S 5$ introduces the class of totally summable functions, for which Fubini's theorem is valid (Theorem 8).

$\S 6$ describes some special properties of integrals of measures (Theorems 9 and 10).

$\S 7$ concerns differentiation (Theorem 11).

Methods. We rely heavily on the deep properties of Suslin spaces expounded in [13]. Although the basic measure space considered in the present article is an abstract measure space, it will be seen that the crucial proofs are accomplished by transporting the measure onto the locally convex Suslin space via the vector function, and then applying the properties of integration in topological spaces to the image measure. The fact that in a Suslin space a bounded measure is ipso facto regular plays an essential role here. In Theorem 5, we consider the following question. Assume a summable function $f$, with values in a locally convex space $F$, takes its values in a subspace $E \hookrightarrow F$, endowed with a stronger topology than the one induced by $F$, and let us suppose the integrals $\int_{A} f d m$ also lie in $E$. Does it then follow that $f$ is an $E$-valued summable function? The answer turns out to be positive if $E$ is a Suslin space.(2) This is a deep theorem, the proof of which relies on the measurability properties of $E$ and on a theorem of R. Christensen [5, p. 246], according to which an additive set function defined on the set of subsets of the integers is countably additive if it is a Borel function

(2) This was proved earlier [14], under the assumption that $E$ is a separable Fréchet space, with different methods. 
of the set (identified with a point in the product space $\{0,1\}^{N}$ ). Christensen's very deep theorem shows that summability of sequences depends not so much on the topology of the space as on the Borel structure. It is possible to deduce from Christensen's theorem the well-known theorem of Orlicz and Pettis [11] according to which for a sequence $\left(x_{n}\right)_{n \in N}$, in a Banach space, to be summable, it is sufficient that for each $A \subset N$ a sum $S_{A}$ exists such that $S_{A}=\Sigma_{n \in A} x_{n}$ in the weak topology. This theorem generalises immediately to locally convex spaces (by plunging them in a product of Banach spaces) and will be applied as such below.

Omissions. Implicit in the study of integrals $\int_{A} f d m$ is the notion of vector valued measure, since the map $A \rightarrow \int_{A} f d m$ is such a measure. Several of the properties mentioned in $\S 1$ (namely II-VI) really concern the measure rather than the function, and could be proved in a more general setting. The converse question (under what conditions does a given vector measure arise from the integration of a vector function) has also been omitted. Elsewhere we have treated this question in some detail [14]. Let us only observe here that locally convex Suslin spaces form a very natural setting for representation theorems of Dunford-Pettis-Phillips type: a map $u: L^{1}(T, m) \rightarrow E$ is (weakly) compact if and only if there exists a measurable function $f: T \rightarrow E$ with conditionally (weakly) compact range such that $u(\varphi)=\int \varphi f d m$ for all $\varphi \in L^{1}(m)$.

Standard notations and assumptions. In this article $T$ denotes a set, $A=$ $\{A\}$ a $\sigma$-algebra of subsets of $T$, and $m$ a $\sigma$-finite positive measure defined on A.( $\left.{ }^{3}\right)$ The characteristic function of a set $A$ is denoted by $\chi_{A}$.

A scalar function $f: T \rightarrow C$ is said to be measurable if $f^{-1}(B) \in A$ for all Borel subsets $B \subset C$. More generally the word measurable always refers to $A$. We do not assume that $A$ necessarily contains all subsets of null sets. The function $f: T \rightarrow \mathrm{C}$ is $m$-integrable if $f$ is measurable and $\int|f| d m<+\infty$.

Let $E$ and $F$ denote locally convex spaces over $\mathbf{R}$ or $\mathbf{C}$ (we assume $\mathbf{C}$ ). These are always assumed to be separated (Hausdorff) and to be quasi-complete (that is, we assume closed bounded subsets of $E$ to be complete). Besides continuous seminorms on $E$ we also consider lower semicontinuous generalized seminorms. These are maps $x \rightarrow|x|_{\beta}$ from $E$ into $[0,+\infty]$ such that (i) $|x+y|_{\beta} \leqslant|x|_{\beta}+|y|_{\beta}$, (ii) $|\lambda x|_{\beta}=|\lambda||x|_{\beta}, \lambda \in \mathbf{C},(0 x+\infty=0)$, (iii) $\beta=$ $\left\{x:|x|_{\beta} \leqslant 1\right\}$ is closed. Every closed set $\beta$ which is absolutely convex (convex

(3) We assume $\sigma$-finiteness to avoid technicalities. But, except in connection with Fubini's theorem, it would suffice to assume that $T$ has a, not necessarily countable, partition $T=\Sigma_{\alpha} T_{\alpha}$ such that (i) $A \in A \Leftrightarrow A \cap T_{\alpha} \in A \forall_{\alpha}$; (ii) $m\left(T_{\alpha}\right)<+\infty$; (iii) $m(A)=$ $\Sigma_{\alpha} m\left(A \cap T_{\alpha}\right)$ for all $A \in A$. 
and balanced: $\lambda \beta \subset \beta$ for $|\lambda| \leqslant 1$ ) is the unit ball of a lower semicontinuous generalised seminorm $x \rightarrow|x|_{\beta}=\inf \{\lambda: x \in \lambda \beta\}$. If $B \subset E$ is closed absolutely convex and bounded, the restriction of $|\cdot|_{B}$ to the linear subspace $E_{B}=\bigcup_{\lambda>0} \lambda B$ $=\left\{x \in E:|x|_{B}<+\infty\right\}$ is a norm. The inclusion map $E_{B} \hookrightarrow E$ is continuous and if $B$ is complete in $E$ (which is always the case if $E$ is quasi-complete) $E_{B}$ is a Banach space.(4) The most important example of a lower semicontinuous seminorm is the norm on the dual of a Banach space equipped with the weak dual topology. If $V$ is a closed absolutely convex neighborhood of zero, $|\cdot|_{V}$ is a continuous seminorm and every continuous seminorm is of this form. Continuous seminorms will also be denoted by $p: x \rightarrow|x|_{p}$. The normed space associated with $p$ is the quotient space $E /{ }_{p^{-1}(0)}$ with the norm $\dot{x} \rightarrow\|\dot{x}\|_{p}=|x|_{p}$ where $x \rightarrow \dot{x}$ is the quotient map. The completion $E_{p}$ of this space is the Banach space associated with $p$.

The dual of $E$ is denoted by $E^{\prime}$ and the duality by $\left\langle x, x^{\prime}\right\rangle$. A subset $H \subset E^{\prime}$ is total if it separates the points of $E$. The polar of a set $S \subset E$ is $S^{\circ}=\left\{x^{\prime} \in E^{\prime}\right.$ : $\left.\left|\left\langle x, x^{\prime}\right\rangle\right| \leqslant 1 \forall x \in S\right\}$. Thus by the Hahn-Banach theorem $|x|_{\beta}=\sup _{x^{\prime} \in \beta^{\circ}}\left|\left\langle x, x^{\prime}\right\rangle\right|$. With the weak dual topology $E^{\prime}$ is denoted by $E_{\sigma}^{\prime}$; with the strong dual topology (uniform convergence on bounded subsets of $E$ ), by $E_{b}^{\prime}$. We also consider $E_{c}^{\prime}$, the dual with the topology of uniform convergence on compact sets.

If $f: T \rightarrow E$ is an $E$-valued function, the function $t \rightarrow\left\langle f(t), x^{\prime}\right\rangle$ is denoted by $\left\langle f(\cdot), x^{\prime}\right\rangle$ or $\left\langle f, x^{\prime}\right\rangle$.

1. Pettis summable functions. Let $E$ be a quasi-complete locally convex Hausdorff space.

Definition 1. A function $f: T \rightarrow E$ is weakly m-summable if $\left\langle f(\cdot), x^{\prime}\right\rangle$ is $m$-integrable for each $x^{\prime} \in E^{\prime}$. The function $f$ is said to be $m$-summable (or Pettis integrable) if $f$ is weakly $m$-summable and if for each set $A \in A$ there exists an element $\int_{A} f d m \in E$ such that $\left\langle\int_{A} f d m, x^{\prime}\right\rangle=\int_{A}\left\langle f, x^{\prime}\right\rangle d m$ for all $x^{\prime} \in E^{\prime}$.

REMARK. If, as will be mostly the case, only one measure is involved, the term "summable" instead of " $m$-summable" will be used. $\int f d m$ stands for $\int_{T} f d m$.

The Pettis integral has the following properties (where $f$ stands constantly for a summable function).

I. (i) If $f_{1}$ and $f_{2}$ are $E$-valued summable functions, $f_{1}+f_{2}$ is summable and $\int_{A} f_{1}+f_{2} d m=\int_{A} f_{1} d m+\int_{A} f_{2} d m$.

(4) If $\left(x_{n}\right)_{n \geqslant 1}$ is a Cauchy sequence in $E_{B}$, it converges to some $x$ in $E$; but if $\left|x_{n}-x_{m}\right|_{B}<\epsilon$ for $n, m>N$, then $\left|x_{n}-x\right|_{B}<\epsilon$ for $n>N, \epsilon B$ being closed in $E$. Hence $x \in E_{B}$ and $x_{n}$ tends to $x$ in $E_{B}$. 
(ii) If $u: E_{1} \rightarrow E_{2}$ is a continuous linear map, and if $f: T \rightarrow E_{1}$ is summable, $u \circ f$ is summable and $\int_{A} u \circ f d m=u \int_{A} f d m$.

II. The map $A \rightarrow \int_{A} f d m$ is countably additive.

III. If $g: T \rightarrow \mathrm{C}$ is bounded and measurable, $g f$ is summable. Moreover, if $V$ is a neighborhood of zero,

$$
\left|\int_{A} f d m\right|_{V} \leqslant \sup _{|g| \leqslant 1}\left|\int_{A} g f d m\right|_{V}=\sup _{x^{\prime} \in V^{\circ}} \int_{A}\left|\left\langle f, x^{\prime}\right\rangle\right| d m<+\infty .
$$

IV. For every neighborhood $V$ of zero there exists a set $A \in A$, with $m(A)<$ $+\infty$, such that $B \in A, B \cap A=\varnothing$ and $|g| \leqslant 1$ imply $\int_{B} g f d m \in V$.

V. The integrals are absolutely continuous:

$$
\lim _{m(A) \rightarrow 0} \int_{A} g f d m=0 \text { uniformly for }|g| \leqslant 1 .
$$

VI. The bounded convergence property: $\left|g_{n}\right| \leqslant 1, g_{n}(t) \rightarrow g(t)$ for almost all $t$ implies $\int g f d m=\lim _{n \rightarrow \infty} \int g_{n} f d m$.

VII. Summability criterion:

(i) If $f(t)=\varphi(t) x$ where $x \in E$ and $\varphi$ is a complex integrable function, $f$ is summable and $\int_{A} f d m=\int_{A} \varphi d m x$.

(ii) If $\left(f_{n}\right)$ is a sequence of summable functions converging weakly in measure to $f_{\infty}$ (i.e. $\left\langle f_{n}, x^{\prime}\right\rangle$ converges in measure to $\left\langle f_{\infty}, x^{\prime}\right\rangle$ for all $x^{\prime}$ ), and the integrals $\int_{A} f_{n} d m$ converge weakly in $E$ for all $A \in A, f_{\infty}$ is summable and $\int_{A} f_{\infty} d m$ is the weak limit of the integrals $\int_{A} f_{n} d m .\left(^{(5)}\right.$

Proof. I is a trivial consequence of the definition and of the fact that $y^{\prime} \circ u \in E_{1}^{\prime}$ for every $y^{\prime} \in E_{2}^{\prime}$.

II follows from the basic Orlicz-Pettis theorem (cf. Introduction) applied to a sequence $x_{n}=\int_{A_{n}} f d m$ where the sets $A_{n}$ are mutually disjoint.

III. To prove the last inequality assume simply that $f$ is weakly summable. Let $E_{V^{\circ}}^{\prime}$ be the Banach space generated in $E^{\prime}$ by $V^{\circ}$ (a compact hence complete set in $\left.E_{0}^{\prime}\right)$. Then the last inequality states that the map $x^{\prime} \rightarrow f_{x^{\prime}}=\left\langle f, x^{\prime}\right\rangle$ from $E_{V^{\circ}}^{\prime}$ to $L^{1}(m)$ is bounded, and this follows immediately from the closed graph theorem. Now since $|x|_{V}=\sup _{x^{\prime} \in V^{\circ}}\left|k x, x^{\prime}\right\rangle \mid$ we have $\left|\int_{A} g f d m\right|_{V} \leqslant$ $\sup _{x^{\prime} \in V^{\circ}} \int\left\langle f f, x^{\prime}\right\rangle \mid d m$ for every simple (i.e., finitely valued) measurable function $g$ with $|g| \leqslant 1$. Thus the map $g \rightarrow \int_{A} g f d m$ from the space of simple functions, with the topology of uniform convergence, to $E$, is continuous and consequently has a continuous extension to the space of all bounded measurable functions. If we denote by $\int_{A}^{*} g f d m$ the value of this extension at $g$, the uniqueness of a

(5) Of course in VI and VII we assume either that A contains all subsets of null sets or that the limit functions are measurable. 
continuous extension implies the relations

$$
\left\langle\int_{A}^{*} g f d m, x^{\prime}\right\rangle=\int_{A} g\left\langle f, x^{\prime}\right\rangle d m=\int_{A}\left\langle g f, x^{\prime}\right\rangle d m
$$

and so $g f$ is summable and $\int_{A} g f d m=\int_{A}^{*} g f d m$. Now

$$
\int_{A}\left\langle f, x^{\prime}\right\rangle\left|d m=\sup _{|g| \leqslant 1}\right| \int_{A} g\left\langle f, x^{\prime}\right\rangle d m \mid
$$

hence

$$
\begin{aligned}
\sup _{x^{\prime} \in V^{\circ}} \int_{A}\left|\left\langle f, x^{\prime}\right\rangle\right| d m & =\sup _{|g| \leqslant 1} \sup _{x^{\prime} \in V^{\circ}}\left|\left\langle\int_{A} g f d m, x^{\prime}\right\rangle\right| \\
& =\sup _{|g| \leqslant 1}\left|\int_{A} g f d m\right|_{V} \geqslant\left|\int_{A} f d m\right|_{V} .
\end{aligned}
$$

IV. We assume $m(T)=+\infty$ since otherwise the condition is vacuous. It is sufficient to prove the assertion when $V$ is closed, and so by the countable additivity it is sufficient to find $A$ for which the property holds with $m(B)<$ $+\infty$. If no such $A$ exists we can find a sequence $\left(A_{n}\right)_{n \in N}$ of disjoint sets of finite measure and functions $g_{n}$ with $\left|g_{n}\right| \leqslant 1$, such that $\int_{A_{n}} g_{n} f d m \notin V$. Let $g=\Sigma_{n} \chi_{A_{n}} g_{n}$. Then $g f$ is summable and $\int g f d m=\Sigma_{n} \int_{A_{n}} g f d m=\Sigma_{n} \int_{A_{n}} g_{n} f d m=$ 0 which implies $\lim _{n \rightarrow \infty} \int_{A_{n}} g_{n} f d m=0$ in contradiction with the construction.

V. By IV it is sufficient to prove this in the case where $m(T)<+\infty$, which we now assume. By III we have to prove that

$$
\lim _{m(A) \rightarrow 0} \sup _{x^{\prime} \in V^{\circ}} \int_{A}\left\langle f, x^{\prime}\right\rangle \mid d m=0
$$

for any neighborhood $V$. Now by II the measures $A \rightarrow \int_{A}\left\langle f, x^{\prime}\right\rangle d m$ are uniformly countably additive for $x^{\prime} \in V^{\circ}$, and so by Theorems IV 9.1 and 9.2 of Dunford and Schwartz [6] they are uniformly absolutely continuous, which proves V.(6)

REMARK. It was demonstrated that the last inequality in III remains valid for arbitrary weakly summable functions.

2. Summable functions with values in Suslin spaces. Recall that a topological space $E$ is a Suslin space if it is Hausdorff and if there exists a Polish space $P$ and a continuous map $\varphi: P \rightarrow E$ onto $E$. Since a Polish space is separable, every Suslin space is separable. Conversely the most common separable linear topologi[6].

(6) Reference to this treatise will henceforth be indicated by the abbreviation D \& $S$ 
cal spaces are Suslin spaces. Separable Banach and Fréchet spaces are Polish, hence Suslin, spaces. The dual $E_{c}^{\prime}$ of a separable Fréchet space is a Suslin space; a fortiori $E_{\sigma}^{\prime}$ is a Suslin space. Any closed subspace of a Suslin space is a Suslin space; a product, hence a projective limit, of a sequence of Suslin spaces is Suslin. A separated quotient of a Suslin space is Suslin; a locally convex direct sum, hence a separated inductive limit, of a sequence of locally convex Suslin spaces is Suslin. The space of operators in a separable Fréchet space $L_{c}(X)$, with the topology of compact convergence, or the "strong operator topology" (pointwise convergence) is a Suslin space. The function spaces $D(\Omega), E(\Omega)$, $S\left(\mathbf{R}^{n}\right)$ and their duals $D^{\prime}, E^{\prime}, S^{\prime}$ with their strong dual topologies are all Suslin spaces (cf. L. Schwartz [13]).

We shall use the following three important properties of Suslin spaces: (1) If $E$ is a Suslin space and $E_{s}$ is the set $E$ equipped with a weaker Hausdorff topology, $E_{s}$ (which incidentally is a Suslin space) has the same Borel sets as $E$. (2) Any finite positive Borel measure $\mu$ on a Suslin is inner regular: $\mu(B)=$ $\sup _{K \subset B} \mu(K), K$ compact. (3) Any family $\left(\varphi_{i}\right)_{i \in I}$ of continuous functions on a Suslin space separating the points of this space has a countable subfamily which still separates the points. In particular if $E$ is a locally convex Suslin space, $E^{\prime}$, and every total subset of $E^{\prime}$, contains a countable total subset. The proofs of these theorems may be found in [13].

From now on we make the permanent assumption that $E$ is a locally convex, quasi-complete, Suslin space.

THEOREM 1. Given a function $f: T \rightarrow E$ with values in a locally convex Suslin space, the following conditions are equivalent:

(a) $f^{-1}(B) \in A$ for all Borel subsets $B \subset E$.

(b) The scalar functions $\left\langle f(\cdot), x^{\prime}\right\rangle$ are measurable for all $x^{\prime} \in E^{\prime}$.

(c) There exists a total subset $H \subset E^{\prime}$ such that $\left\langle f(\cdot), x^{\prime}\right\rangle$ is measurable for all $x^{\prime} \in H .\left({ }^{7}\right)$

Proof. Obviously (a) implies (b) which implies (c). To prove that (c) implies (a) we may assume that $H$ is countable, because $H$ contains a countable total subset. Using the injection $x \rightarrow\left(\left\langle x, x^{\prime}\right\rangle\right)_{x^{\prime} \in H}$, consider $E$ as a linear subspace of $\mathbf{C}^{H}$. It is known that the Borel $\sigma$-algebra of $\mathbf{C}^{H}$ is the smallest for which the coordinate projections are measurable. Also, $E$ being a Suslin space, the Borel subsets of $E$ are the same as the Borel subsets of $E$ equipped with the topology induced by $\mathbf{C}^{H}$. This completes the proof.

Remark . If $E$ is a Lusin space (continuous injective image of a Polish

(7) This is merely a refinement of the equivalence (a) $\Leftrightarrow(b)$, the proof of which may be found in [13]. 
space), (a) (b) (c) are equivalent to:

(d) There exists a sequence $\left(f_{n}\right)_{n \in N}$ of measurable simple functions such that $f(t)=\lim _{n \rightarrow \infty} f_{n}(t)$ for all $t$.

Proof. Since there is a Polish topology, with the same Borel sets stronger than the given topology on $E$, (a) implies (d). Also (d) implies (b).

We shall not use this remark hereafter.

Definition 2. When the conditions of Theorem 1 are met, we shall say that $f$ is a measurable function.

Thus by condition (b) every $m$-summable function with values in a locally convex Suslin space is measurable. We shall now state further properties of an $m$-summable function with values in a quasi-complete locally convex Suslin space E.

VIII. Let $E_{+} \subset E$ be a closed convex cone in $E$ (or, in particular, a closed subspace). Then $\int_{A} f d m \in E_{+}$for all $A \in A$ if and only if $f(t) \in E_{+} m$ a.e.

Also, if $f_{1}$ and $f_{2}$ are summable $\int_{A} f_{1} d m=\int_{A} f_{2} d m$ for all $A$ if and only if $f_{1}(t)=f_{2}(t)$ a.e.

IX. Let $x \rightarrow|x|_{\beta}$ be a lower semicontinuous generalized seminorm on $E$. Then $|f(\cdot)|_{\beta}$ is measurable and $\left|\int_{A} f d m\right|_{\beta} \leqslant \int_{A}|f|_{\beta} d m \leqslant+\infty$.

X. Let $\mathrm{m}(A)=\int_{A} f d m$ and $|\mathrm{m}|_{\beta}(A)=\sup _{\Sigma A_{i} \subset A} \sum\left|\mathrm{m}\left(A_{i}\right)\right|_{\beta}$ (supremum over all finite systems of mutually disjoint measurable subsets of $A$ ). Then

$$
\int_{A}|f|_{\beta} d m=|\mathrm{m}|_{\beta}(A)
$$

Equivalently,

$$
\int_{A}|f|_{\beta} d m=\sup _{|\varphi|_{\beta}^{\circ} \leqslant 1}\left|\int_{A}\langle f(t), \varphi(t)\rangle d m(t)\right|,
$$

where the supremum is taken over all measurable simple functions $\varphi=\Sigma \chi_{A_{i}} x_{i}^{\prime}$ with values in $\beta^{\circ}$.

Proof OF VIII. Let $E_{+}^{\prime}$ be the set of real, continuous R-linear forms such that $\left\langle x, x^{\prime}\right\rangle \geqslant 0$ for all $x \in E_{+}$. Then by the Hahn-Banach theorem $E_{+}=$ $\left\{x:\left\langle x, x^{\prime}\right\rangle \geqslant 0 \forall x^{\prime} \in E_{+}^{\prime}\right\}$. If $f(t) \in E_{+}$a.e., then $\left\langle\int_{A} f d m, x^{\prime}\right\rangle=$ $\int_{A}\left\langle f(t), x^{\prime}\right\rangle d m(t) \geqslant 0$ for all $x^{\prime} \in E_{+}^{\prime}$, and so $\int_{A} f d m \in E_{+}$. Conversely $\int_{A} f d m \in E_{+}$for all $A$ implies $\left\langle f(t), x^{\prime}\right\rangle \geqslant 0$ a.e., but the exceptional set depends a priori on $x^{\prime}$. When $E_{+}$is a closed subspace this difficulty can be overcome immediately by observing that the quotient $E / E_{+}$is a Suslin space, so that $\left(E / E_{+}\right)^{\prime}$ contains a countable total subset. Similarly the last assertion in VIII is obvious because $E^{\prime}$ contains a countable total subset. The proof in the general case of a cone results from the following proposition which also has other applications: 
Localization Lemma. Let $S$ be a closed convex subset of $E$ and let $f: T \rightarrow E$ be a measurable function such that for every closed half space $D$ containing $S, f(t) \in D$ a.e. Then $f(t) \in S$ a.e.

Proof. It is sufficient to prove this when $m(T)<+\infty$. Let $\mu$ be the Borel measure on $E$ defined by $\mu(B)=m\left(f^{-1}(B)\right)$. Then, since $E$ is a Suslin space, $\mu$ is inner regular; in particular $\mu$ has a support, the complement of the largest open null set. Now by hypothesis $\mu(C D)=0$ and so $\operatorname{supp}(\mu) \subset D$. By the Hahn-Banach theorem $S$ is the intersection of all the closed half spaces $D$ containing $S$, and consequently $\operatorname{supp}(\mu) \in S$. A fortiori $m\left(\mathbf{C} f^{-1}(S)\right)=\mu(\mathbf{C} S)=0$.

Proof of IX. Since $|\cdot|_{\beta}$ is lower semicontinuous, $|\cdot|_{\beta}$ is Borel measurable and so $|f(\cdot)|_{\beta}$ is measurable by Theorem 1 . Also

$$
\left|\int_{A} f d m\right|_{\beta}=\sup _{x^{\prime} \in \beta^{\circ}}\left|\int_{A}\left\langle f, x^{\prime}\right\rangle d m\right| \leqslant \int_{A}|f|_{\beta} d m .
$$

Proof of X. Since $|\mathrm{m}(A)|_{\beta}=\sup _{x^{\prime} \in \beta^{\circ}}\left|\int_{A}\left\langle f, x^{\prime}\right\rangle d m\right|$, the last statement in $X$ is easily seen to be equivalent to the first. Since, by IX,

$$
\sum\left|\mathrm{m}\left(A_{i}\right)\right|_{\beta} \leqslant \sum \int_{A_{i}}|f|_{\beta} d m \leqslant \int_{A}|f|_{\beta} d m,
$$

the inequality $|\mathrm{m}|_{\beta}(A) \leqslant \int_{A}|f|_{\beta} d m$ is obvious. It is not hard to see that $|\mathrm{m}|_{\beta}(\cdot)$ is countably additive, and so it is sufficient to prove the inequality $\int_{A}|f|_{\beta} d m \leqslant|\mathrm{~m}|_{\beta}(A)$ in case $A=T$ with $m(T)<+\infty$. Now let $\mu(B)=$ $m\left(f^{-1}(B)\right)$. Then

$$
\int|f|_{\beta} d m=\int|x|_{\beta} d \mu(x)
$$

and

$$
\mu(B)=\int_{B} x d \mu(x)=\int_{f^{-1}(B)} f d m=\mathrm{m}\left(f^{-1}(B)\right)
$$

Thus $|\mu|_{\beta}(E) \leqslant|\mathrm{m}|_{\beta}(T)$. Consequently, it is sufficient to prove the desired inequality for $\mu$ instead of $m$. The proof of this, which uses the regularity of $\mu$ to reduce the problem to the case where $\mid I_{\beta}$ is finite and continuous, has been given elsewhere [14] in more detail and will not be repeated here. $\left({ }^{8}\right)$

To terminate this section, we consider an example introduced by Graves [7] and used since then by other authors $([1],[11])$ as a counterexample. $\left({ }^{9}\right)$

(8) The proof can be simplified by observing that in the Suslin space $E, \mid \mathrm{I}_{\beta}$ is the upperbound of a sequence of finite continuous seminorms. (Added in proof May 7, 1975.)

(9) I am indebted to D. R. Lewis for pointing out this example to me. 
Example. Let $T$ be the interval $[0,1], A$ the set of Lebesgue measurable subsets of $T, m$ Lebesgue measure, denoted by $d t$. First let $E$ be the space $L^{\infty}[0,1]$ with the weak dual topology $\sigma\left(L^{\infty}, L^{1}\right)$, for which it is a quasi-complete Suslin space. Let $f(t)=\chi_{[t, 1]}$. Then it is easy to see (using Fubini's theorem) that $f$ is summable and that for a bounded measurable function $g$, the integral $\int g f d t$ is just the primitive function $s \rightarrow \int_{0}^{s} g(t) d t$. Now the integral $\int_{0}^{1} f d t$ is the limit in the topology of the norm in $L^{\infty}$, of Riemann sums $\Sigma_{i=1}^{n} f\left(t_{i}\right)\left(t_{i}-t_{i-1}\right)$, $0=t_{0}<t_{i}<t_{n}=1$. From this we can deduce that for an arbitrary linear form in $\left(L^{\infty}\right)^{\prime}$ (continuous in the norm topology), $\int_{A}\left\langle f, x^{\prime}\right\rangle d t=$ $\left\langle\left\langle_{A} f d t, x^{\prime}\right\rangle\right.$. Therefore $f:[0,1] \rightarrow L^{\infty}$ is Pettis summable, even if $L^{\infty}$ is given the norm topology. For every measurable set $A, \int_{A} f d t$ belongs to the closed subspace $C[0,1]$. But $f(t) \notin C[0,1]$ for $t \in(0,1]$.

Thus VIII does not hold without strong measurability assumptions. Neither do the equalities in $X$ hold for arbitrary summable functions without measurability condition. Indeed VIII is a consequence of $X$ in the case of a closed subspace $F=E_{+}$because if we take $\beta=F$, we have $|x|_{\beta}=0$ for $x \in F$, and $|x|_{\beta}=+\infty$ for $x \notin F$. Thus in Graves' example with $\beta=F=C[0,1],|\mathrm{m}|(T)=0,|f(\cdot)|_{\beta}$ is measurable and $\int_{A}|f|_{\beta} d m=+\infty$ for every set $A$ with $m(A)>0$.

3. Summability criteria. Throughout this section we assume that $E$ is a locally convex quasi-complete Suslin space.

Lemma A . Let $T_{1} \in A$ with $m\left(T_{1}\right)<+\infty$. Assume $f: T_{1} \rightarrow E$ is measurable and $f\left(T_{1}\right)$ is conditionally weakly compact. Then $f$ is $m$-summable over $T_{1}$.

Proof (Bourbaki). Let $B$ be the closed absolutely convex hull of $f\left(T_{1}\right)$. By the theorem of Kreĭn-Šmulian (D \& S [6, V.6.4]) generalized to quasicomplete locally convex spaces, $B$ is weakly compact.(10) Also, $f$ being bounded and measurable is weakly summable, and the weak integral, defined by the formula $\left\langle\int_{A} f d m, x^{\prime}\right\rangle=\int_{A}\left\langle f, x^{\prime}\right\rangle d m$, is an element of the weak completion of $E$, identified with the space $E^{\prime *}$ of all linear forms on $E^{\prime}$. If $x^{\prime} \in B^{\circ}$, $\left\langle\int_{A} f d m, x^{\prime}\right\rangle \mid \leqslant \int_{T_{1}} d m$ and so $\int_{A} f d m \in \int_{T_{1}} d m B^{\circ \circ}$, where $B^{\circ \circ}$ is the polar of $B^{\circ}$ in $E^{\prime *}$. But $B$ is weakly compact in $E^{\prime *}$, and so $B^{\circ \circ}=B$ and $\int_{A} f d m \in E$.

LEMma B. Let $f: T \rightarrow E$ be a measurable function. Then there exists a partition $T=\Sigma_{n=0}^{\infty} T_{n}$ of $T$ into measurable subsets, such that $m\left(T_{0}\right)=0$,

(10) The closed absolutely convex hull of a set $K$ is the closed convex hull of $U_{|\lambda| \leqslant 1} \lambda K$. But this set is the image of $\{\lambda:|\lambda| \leqslant 1\} \times K$ under the continuous map $(\lambda, x) \rightarrow \lambda x$ and so (weakly) compact if $K$ is weakly) compact. For the intended application it is sufficient to assume $f\left(T_{1}\right)$ relatively compact, and then the more elementary theorem of Mazur (D \& S V. 2.6) is sufficient. 
$m\left(T_{n}\right)<+\infty$ and $f\left(T_{n}\right)$ is conditionally compact for $n \geqslant 1$.

Proof. We first subdivide $T$ into sets of finite measure. Then it will be sufficient to prove the lemma for each of these, and so we may assume $m(T)<$ $+\infty$. Let $\mu(B)=m\left(f^{-1}(B)\right)$. Then $\mu$ is a finite inner regular measure on $E$, and so $E$ has a partition $E=N+\Sigma_{n=1}^{\infty} K_{n}$, where $K_{n}$ is compact and $\mu(N)=0$. If we put $T_{0}=f^{-1}(N)$ and $T_{n}=f^{-1}\left(K_{n}\right)$, the partition $T=\Sigma_{n=0}^{\infty} T_{n}$ has the required property. $\left({ }^{11}\right)$

THEOREM 2. A measurable function $f: T \rightarrow E$ is m-summable if and only if for every sequence of disjoint sets $T_{n} \in A$, with $m\left(T_{n}\right)<+\infty$, and such that $f\left(T_{n}\right)$ is conditionally compact, the sums $\Sigma_{n} \int_{T_{n}} f d m$ are convergent in $E$.

PROof. The condition is necessary by the countable additivity of the integral. To prove the sufficiency, let $T=\sum_{n=0}^{\infty} T_{n}$ be a partition as in Lemma B. Let $x^{\prime} \in E^{\prime}$ and $T^{+}=\left\{t \in T: \operatorname{Re}\left\langle f(t), x^{\prime}\right\rangle \geqslant 0\right\}$. Then

$$
\begin{aligned}
\int_{T} \operatorname{Re}\left\langle f, x^{\prime}\right\rangle^{+} d m & =\sum_{n=1}^{\infty} \int_{T}+\cap T_{n} \operatorname{Re}\left\langle f, x^{\prime}\right\rangle d m \\
& =\sum_{n=1}^{\infty} \operatorname{Re}\left\langle\int_{T}{ }^{+\cap T_{n}} f d m, x^{\prime}\right\rangle<+\infty .
\end{aligned}
$$

Similarly the other positive components of $\left\langle f, x^{\prime}\right\rangle$ are seen to be integrable, and so $f$ is weakly summable. Now put $\int_{A}^{*} f d m=\sum_{n=1}^{\infty} \int_{A \cap T_{n}} f d m$. Then

$$
\begin{aligned}
\left\langle\int_{A}^{*} f d m, x^{\prime}\right\rangle & =\sum_{n=1}^{\infty}\left\langle\int_{A \cap T_{n}} f d m, x^{\prime}\right\rangle \\
& =\sum_{n=1}^{\infty} \int_{A \cap T_{n}}\left\langle f, x^{\prime}\right\rangle d m=\int_{A}\left\langle f, x^{\prime}\right\rangle d m
\end{aligned}
$$

Thus $f$ is summable and $\int_{A}^{*} f d m=\int_{A} f d m$.

REMARK 1. In the proof we only used the weak convergence of the sums. Since weak and strong convergence of the sums are equivalent by Orlicz's theorem this is not surprising.

RemarK 2. Property VII ( $\S 1$ ), could have been used in the proof; however, the present proof is more elementary than VII and is also valid under the more general assumption on $m$ (note that a family of elements in $E$ is summable if each countable subfamily is summable, so that the statement of Theorem 2 remains valid under the more general assumption on $m$ ).

(11) In this statement we assumed that $m$ is $\sigma$-finite. If the more general hypothesis is adopted (footnote 2) the lemma asserts the existence of partition as there described with the properties of Lemma B. The proof is the same. 
THEOREM 3. Let $f: T \rightarrow E$ be a measurable function such that $\int|f|_{p} d m<$ $+\infty$ for every continuous seminorm $p$. Then $f$ is m-summable.

Proof. Let $\left(T_{n}\right)_{n \geqslant 1}$ be a sequence as in the statement of Theorem 2. Then

$$
\sum_{n}\left|\int_{T_{n}} f d m\right|_{p} \leqslant \sum_{n} \int_{T_{n}}|f|_{p} d m \leqslant \int|f|_{p} d m<+\infty,
$$

and so $\Sigma_{n} \int_{T_{n}} f d m$ converges in $E$ by Cauchy's criterion.

Definition 3. Functions $f: T \rightarrow E$ satisfying the conditions of Theorem 3 are said to be absolutely m-summable.

COROLlaRY 3.1. If $m(T)<+\infty$ and $f: T \rightarrow E$ is bounded and measurable, $f$ is absolutely summable.

REMARK . Now that we know this, we can also apply Theorem 2 with sequences $\left(T_{n}\right)$ such that $m\left(T_{n}\right)<+\infty$, and such that $f\left(T_{n}\right)$ is merely bounded.

Definition 4. A closed convex cone $E_{+} \subset E$ is said to be weakly $\Sigma$-complete if for every sequence $\left(x_{n}\right)_{n \in N}$ of points of $E_{+}$, for which $\Sigma_{n}\left|x_{n}, x^{\prime}\right\rangle \mid<$ $+\infty$ for all $x^{\prime}$, there is $x \in E_{+}$such that $\left\langle x, x^{\prime}\right\rangle=\Sigma_{n}\left\langle x_{n}, x^{\prime}\right\rangle$. (In which case the sum $\Sigma x_{n}$ converges strongly in $E$ by Orlicz's theorem.) .

THEOREM 4. Let $f: T \rightarrow E_{+}$be a function with values in a weakly $\Sigma$-complete cone. Then if $f$ is weakly m-summable, $f$ is m-summable.

Proof. By Theorem $1 f$ is measurable. Let $\left(T_{n}\right)_{n \geq 1}$ be a sequence as in Theorem 2. Then $\int_{T_{n}} f d m \in E_{+}$and

$$
\sum_{n}\left|\left\langle\int_{T_{n}} f d m, x^{\prime}\right\rangle\right| \leqslant \sum_{n} \int_{T_{n}}\left|\left\langle f, x^{\prime}\right\rangle\right| d m \leqslant \int\left|\left\langle f, x^{\prime}\right\rangle\right| d m<+\infty,
$$

and so $f$ is summable by Theorem 2 .

COROLLARY 4.1. If the space $E$ is weakly sequentially complete, in particular if $E=E^{\prime \prime}$, every weakly summable function is summable.

Proof. If $E$ is weakly sequentially complete, i.e. if every weak Cauchy sequence is weakly convergent, $E$ is a fortiori weakly $\Sigma$-complete. If $E$ is semireflexive, i.e. if the canonical map $E \rightarrow E^{\prime \prime}$ is onto, the weakly closed bounded subsets of $E$ are weakly compact, hence weakly complete; in particular every weak Cauchy sequence converges weakly.

Remark . As always we assume that $E$ is quasi-complete and Suslin. A semireflexive space is always quasi-complete (since a weakly complete subset of $E$ is complete). 
THEOREM 5. Let $E$ and $\widetilde{E}$ be quasi-complete locally convex Hausdorff spaces, $E$ being a Suslin space. Assume $E$ is a linear subspace of $\widetilde{E}$ such that the inclusion $j: E \hookrightarrow \widetilde{E}$ is continuous. Let $f: T \rightarrow E$ be an $E$-valued function and put $\tilde{f}=j \circ f$. Then $f$ is $m$-summable if and only if the following conditions are satisfied: $\widetilde{f}$ is $m$-summable and $\int_{A} \widetilde{f} d m \in E$ for all $A \in A$.

Corollary 5.1. Let $H \subset E^{\prime}$ be any total subset of $E^{\prime}$. Let $f: T \rightarrow E$ be a function such that $(1)\left\langle f(\cdot), x^{\prime}\right\rangle$ is integrable for all $x^{\prime} \in H$, and (2) associated with each $A \in A$ there is an element $I_{A} \in E$ such that $\left\langle I_{A}, x^{\prime}\right\rangle=\int_{A}\left\langle f, x^{\prime}\right\rangle d m$ for all $x^{\prime} \in H$. Then $f$ is $m$-summable and $I_{A}=\int_{A} f d m$.

Proof. The corollary follows from the theorem by plunging $E$ into $\mathrm{C}^{H}$ via the map $x \rightarrow\left(\left\langle x, x^{\prime}\right\rangle\right)_{x^{\prime} \in H^{\prime}}$.

The condition stated in the theorem is necessary by property I(ii). To prove the sufficiency we first consider the particular case of a discrete measure. Let $x_{n} \in E$ and assume $\left(x_{n}\right)_{n \in N}$ is summable in $\widetilde{E}$ and that the sums $s(A)=$ $\Sigma_{n \in A} x_{n}$ (strictly $\Sigma_{n \in A} \tilde{x}_{n}$ ) belong to $E$. Let $E_{s}$ be the space $E$ with the topology induced by $\widetilde{E}$. Then the map $\chi_{A} \rightarrow s(A)$ from $\{0,1\}^{N}$, equipped with the product topology, to $E_{s}$, is continuous (a special case of the bounded convergence property). Also the identity map $E_{s} \rightarrow E$ is a Borel map, since $E$ is a Suslin space. Therefore $\chi_{A} \rightarrow s(A) \in E$ is a Borel map and for every $x^{\prime} \in E^{\prime}, \chi_{A} \rightarrow$ $\left\langle s_{A}, x^{\prime}\right\rangle$ is a Borel map. It follows from the theorem of $\mathrm{R}$. Christensen [5, Theorem 2], that $A \rightarrow\left\langle s(A), x^{\prime}\right\rangle$ is countably additive, and consequently $\left(x_{n}\right)_{n \in N}$ is summable in $E$ by Orlicz's theorem. $\left({ }^{12}\right)$

We now turn to the proof of Theorem 5 in the general case. The restrictions to $E$ of the continuous linear forms on $\widetilde{E}$, separate the points of $E$, and so by Theorem $1, f$ is measurable. Let $\left(T_{n}\right)_{n \in N}$ be a sequence of measurable mutually disjoint subsets of $T$, with $m\left(T_{n}\right)<+\infty$, and $f\left(T_{n}\right)$ conditionally compact in $E$. For $P \subset N$ put $T_{P}=\Sigma_{n \in P} T_{n}$. Then the sequence $\left(\int_{T_{n}} f d m\right)_{n}=\left(\int_{T_{n}} \widetilde{f} d m\right)_{n}$ is summable in $\widetilde{E}$ and the sums $\Sigma_{n \in P} \int_{T_{n}} \widetilde{f} d m=\int_{T_{p}} \widetilde{f} d m$ belong to $E$ by hypothesis. Thus $\left(\int_{T_{n}} f d m\right)_{n}$ is summable in $E$ and $f$ is summable by Theorem 2 .

Example. Let $\left(T_{t}\right)_{t}$ be a family of distributions in an open subset $\Omega$ of $\mathbf{R}^{n}$ and assume the function $t \rightarrow\left\langle T_{t}, \varphi\right\rangle$ is integrable for each $\varphi \in D(\Omega)$. Then $t \rightarrow T_{t}$ is summable (Corollary 4.1). Put $T_{A}=\int_{A} T_{t} d m(t)$. Assume it is known that the distributions $T_{t}$ and $T_{A}$ all belong to one of the following spaces: $D(\Omega)$, $D^{(m)}(\Omega), L_{\text {loc }}^{1}(\Omega), L^{p}(\Omega)(1 \leqslant p<+\infty), L^{\infty}(\Omega)_{\sigma}, D^{(m)}(\Omega)_{\sigma}^{\prime}, M(\Omega)=C_{0}(\Omega)_{\sigma}^{\prime}$.

(12) In a subsequent paper Christensen proves the validity of his theorem for additive set functions with values in arbitrary separable topological groups. So the above use of linear forms and Orlicz's theorem is really unnecessary. On the contrary, Orlicz's theorem could be deduced from Christensen's theorem. 
Then $t \rightarrow T_{t}$ is summable as function with values in $D(\Omega), D^{(m)}(\Omega)$ etc. valued function. It is not sufficient to assume merely that the total integral $\int T_{t} d m(t)$ belongs to the subspace in question. For instance let $T_{t}$ be the locally integrable function $x \rightarrow e^{2 \pi i x t}$, with $\Omega=\mathbf{R}$. Then we have in $D^{\prime}(\mathbf{R})$ or $S^{\prime}(\mathbf{R}), \delta=\int e^{2 \pi i \cdot t} d t$, where $\delta$ is the Dirac measure at 0 . The integrand and the integral belong to the space of Radon measures (or temperate Radon measures). But

$$
\int_{0}^{+\infty} e^{2 \pi i \cdot t} d t=\frac{1}{2} \delta-\frac{1}{2 \pi i} p v \frac{1}{x}
$$

which is not a measure.

The integrals $\int_{A} T_{t} d m(t)$ may also be "more regular" than the distributions $T_{t}$, but it can be shown (cf. [14]) that if for each $A$ the integral $\int_{A} T_{t} d m(t)$ belongs to $E=D(\Omega), E(\Omega), S^{\prime}\left(\mathrm{R}^{n}\right)$, we necessarily also have $T_{t} \in E$ a.e. If $\int_{A} T_{t} d m(t) \in \mathrm{C}(\Omega)$, the space of continuous functions, $T_{t} \in L_{\text {loc }}^{1}(\Omega)$ a.e. More obvious is the fact that if the integrals $\int_{A} T_{t} d m(t)$ are all positive measures, the $T_{t}$ are almost all positive measures. This follows from property VIII and the fact that the cone of positive measures is closed in $D^{\prime}(\Omega)$. In that case $\left\langle T_{A}, \varphi\right\rangle=$ $\int_{A}\left\langle T_{t}, \varphi\right\rangle d m(t)$ for all continuous functions with compact support, $\varphi$, and moreover, for all bounded Borel functions with compact support (see $\S 6$ ).

REmark. Theorem 5 also holds for an arbitrary reflexive Banach space $E$ (and the proof is trivial since a total subspace of $E^{\prime}$ is dense in the norm topology). A complete discussion of nonseparable reflexive Banach spaces does not fit in the framework of this article. It has been shown by Grothendieck that if $f$ : $T \rightarrow E$ is a summable function on a $\sigma$-finite measure space, with values in a reflexive Banach space, there exists a summable function $f_{1}: T \rightarrow E_{1}$, where $E_{1}$ is a separable closed subspace, such that $\int_{A} f d m=\int_{A} f_{1} d m$ for all $A$.

4. Nuclear spaces and Bochner integrable functions. Let $E$ and $F$ be locally convex spaces. Recall that a continuous linear map $u: E \rightarrow F$ is nuclear if it has an expansion of the form

$$
u(x)=\sum_{n=1}^{\infty} \lambda_{n}\left\langle x, x_{n}^{\prime}\right\rangle y_{n}
$$

where $\lambda_{n} \in \mathrm{C}$ with $\Sigma\left|\lambda_{n}\right|<+\infty, x_{n}^{\prime} \in V^{\circ}$, for some neighborhood of zero $V$, in $E$, and $y_{n} \in B$, where $B$ is a closed bounded absolutely convex set such that $F_{B}$ is complete. A locally convex space $E$ is nuclear if for every continuous seminorm $p$, the canonical map $E \rightarrow E_{p}$, to the Banach space associated with $p$, is a nuclear map. It then follows that every continuous linear map $u: E \rightarrow F$ to a Banach space is nuclear (since for some $p$ it factorizes $E \rightarrow E_{p} \rightarrow F$ ). Sub- 
spaces, products, quotient spaces of nuclear spaces are nuclear, and the inductive limit of a sequence of nuclear spaces is nuclear. A Fréchet space is nuclear if and only if its strong dual is nuclear. (See Grothendieck [9, pp. 39, 40].) A very useful criterion for nuclearity was given by A. Pietsch [12, p. 64]. The spaces $D, D^{\prime}, E, E^{\prime}, S, S^{\prime}$, are nuclear spaces (and incidentally quasi-complete Suslin spaces). Every locally convex space equipped with its weak topology is trivially nuclear.

THEOREM 6. Let $E$ be a quasi-complete nuclear Suslin space. Then every weakly summable function $f: T \rightarrow E$ is absolutely summable.

Proof. Let $p$ be a continuous seminorm on $E$. The canonical map $E \rightarrow$ $E_{p}$ is nuclear, hence $x^{2}=\Sigma \lambda_{n}\left\langle x, x_{n}^{\prime}\right\rangle y_{n}$ with $\left\|y_{n}\right\|_{p} \leqslant 1, \Sigma\left|\lambda_{n}\right|<+\infty, x_{n}^{\prime} \in V^{\circ}$. A fortiori $|x|_{p} \leqslant \Sigma_{n}\left|\lambda_{n}\right|\left\langle x, x_{n}^{\prime}\right\rangle$. If $M=\sup _{x^{\prime} \in V^{\circ}} \int\left|\left\langle f, x^{\prime}\right\rangle\right| d m$ (cf. Remark at end of $\S 1$ ), then

$$
\int|f|_{p} d m \leqslant M \sum_{n}\left|\lambda_{n}\right|<+\infty
$$

REMARK. If $E$ is a Fréchet space and if $m$ is not a finite sum of point masses the above property holds only if $E$ is nuclear (cf. [12, 4.2.5]).

Many nuclear spaces are also duals of nuclear spaces, and for some of these a much stronger statement can be made, after the following definition.

Definition 5. A function $f: T \rightarrow E$ will be said to be Bochner integrable if there exists a Banach subspace $E_{B} \hookrightarrow E$ such that $f(t) \in E_{B}$ a.e. (i.e. for $t \in T_{0} \in A$ with $m\left(T-T_{0}\right)=0$ ) and such that $f: T_{0} \rightarrow E_{B}$ is Bochner integrable in the established sense. $\left({ }^{13}\right)$

If $f$ is Bochner integrable it follows that $f: T \rightarrow E$ is measurable (Definition 2) and hence absolutely $m$-summable. The Bochner integrals $\int_{A} f d m$ in $E_{B}$ coincide with the integrals of $f$ as $E$-valued summable function.

THEOREM 7. Let E be a Suslin space, dual of a quasi-complete barrelled nuclear space $F$. Then every weakly summable function $f: T \rightarrow E$ is Bochner integrable.

Proof. There is only an apparent ambiguity in the statement because $F_{b}^{\prime}$ and $F_{\sigma}^{\prime}$ have the same dual (namely $F$ ) and the same bounded sets. $F$ is semireflexive andbarrelled hence reflexive, and $f$ is summable by Corollary $4.1 .\left(^{14}\right)$ We denote by $x$ the elements of $F$. Let

(13) That is $f: T_{0} \rightarrow E_{B}$ is strongly measurable (limit a.e. of a sequence of simple functions), and $\int|f|_{B} d m<+\infty$. When $m$ is $\sigma$-finite this means there is a separable closed subspace $E_{0}$ of $E_{B}$, such that $f(t) \in E_{0}$ for $t \in T_{0}$ with $m\left(T-T_{0}\right)=0$, and $f: T_{0} \rightarrow E_{0}$ is absolutely summable.

(14) We use the terminology and theorems of Bourbaki [3, §3]. 


$$
p(x)=\int|\langle f, x\rangle| d m=\sup _{|g| \leqslant 1} \mid\left\langle\int g f d m, x\right\rangle .
$$

Then $p$ is a seminorm on $F$ and $\{x: p(x) \leqslant 1\}$ is closed, and consequently a barrel, and so by hypothesis a neighborhood of zero in $F$. Thus the map $x \rightarrow$ $f_{x}=\langle f(\cdot), x\rangle$ from $F$ to $L^{1}(m)$ is continuous, and therefore nuclear. Consequently $f_{x}=\Sigma_{n} \lambda_{n}\left\langle x, x_{n}^{\prime}\right\rangle f_{n}$ where $\Sigma\left|\lambda_{n}\right|<+\infty, x_{n}^{\prime} \in B=V^{\circ} \subset E, V$ being a neighborhood of zero in $F$, and $f_{n} \in L^{1}(m)$ with $\int\left|f_{n}\right| d m \leqslant 1$. The series $\Sigma_{n} \lambda_{n} f_{n}(\cdot) x_{n}^{\prime}$ converges absolutely in $L^{1}\left(m ; E_{B}\right)$ and therefore almost everywhere. If $\bar{f}(t)$ is equal to the sum almost everywhere, $\bar{f}: T \rightarrow E_{B}$ is Bochner integrable and

$$
\langle\bar{f}(t), x\rangle=\sum_{n} \lambda_{n} f_{n}(t)\left\langle x, x_{n}^{\prime}\right\rangle=\langle f(t), x\rangle \text { a.e. }
$$

Consequently $f(t)=\bar{f}(t)$ a.e. $\left(F\right.$ is separable since $E^{\prime}$ contains a countable total subset). This ends the proof.

COROLLARY 7.1. Let $E$ be a nuclear Fréchet space. Then every weakly summable function $f: T \rightarrow E$ is Bochner integrable.

Proof . $E$ is separable hence Suslin. Also $E$ is reflexive and so $E_{b}^{\prime}$ is reflexive hence barelled (cf. $\left[3, \S 3, \mathrm{n}^{\circ} 3\right]$ ), and $E_{b}^{\prime}$ is nuclear $[9, \mathrm{p} .40]$. Finally $E=E^{\prime \prime}=$ $\left(E_{b}^{\prime}\right)_{b}^{\prime}$.

The corollary was proved by Grothendieck, who proved that if $E$ is a separable Fréchet space any absolutely summable function is Bochner integrable $[8, \mathrm{p} .68$, Proposition 18], and that if $E$ is nuclear a weakly summable function is absolutely summable $[9$, p. 39, Corollary 5].

EXAMPLE. Every weakly summable function with values in $0, D^{\prime}, E, E^{\prime}, S, S^{\prime}$, is Bochner integrable.

REMARK. In Theorem 7 neither the hypothesis that $F$ is nuclear, nor that $F$ is barrelled can be omitted. For instance, take $F$ to be a separable Hilbert space. If $F$ has the weak topology $F$ is nuclear, quasi-complete, but not barrelled. If $F$ has the norm topology $F$ is complete, barrelled, but not nuclear. In either case $F_{b}^{\prime}=E$ is a separable Hilbert space with the norm topology and there are clearly summable functions (even sequences) which are not Bochner integrable, i.e. absolutely summable.

In practice it may not be easy to determine a closed bounded absolutely convex set $B$ such that $f: T \rightarrow E_{B}$ (defined a.e.) is Bochner integrable. However, without constructing the set $B$ or the space $E_{B}$ explicitly, we may draw the following conclusions:

(1) It is possible to find a sequence of simple functions $\left(f_{n}\right)_{n \in N}$ converging 
to $f$ almost everywhere such that $\int_{A} f d m=\lim _{n \rightarrow \infty} \int_{A} f_{n} d m$. (We can even write $f$ as a series of such functions.)

(2) Fubini's theorem is valid.

(3) For integrals in Euclidean space, the function is equal almost everywhere to the derivative of its integral.

These last two properties can be demonstrated under less restrictive conditions (see $\S \S 5$ and 7).

\section{Totally summable functions: Fubini's theorem.}

DEFinition 6. A measurable function $f: T \rightarrow E$ is said to be totally m-summable if there exists a closed bounded absolutely convex set $B \subset E$ such that $\int|f|_{B} d m<+\infty .\left({ }^{15}\right)$

If $p$ is a continuous seminorm and $M=\sup _{x \in B}|x|_{p}$, then $|x|_{p} \leqslant M|x|_{B}$ for all $x$, whence $\int|f|_{p} d m \leqslant M \int|f|_{B} d m<+\infty$. Thus every totally summable function is absolutely summable.

Let us recapitulate the types of summability considered so far. These are the following, each of which is a more restrictive concept than the next:

(1) $f$ is Bochner integrable.

(2) $f$ is totally summable.

(3) $f$ is absolutely summable.

(4) $f$ is summable (or Pettis integrable).

(5) $f$ is weakly summable.

A function $f$ with values in the weak dual $E_{\sigma}^{\prime}$ of a separable Banach space $E$ is totally summable if and only if it is weakly measurable (i.e. $\langle f(\cdot), x\rangle$ is measurable for each $x \in E$ ) and $\int\|f(t)\| d m(t)<+\infty$. It is a Bochner integrable function if, furthermore, $f$ is strongly measurable (i.e. a.e. limit in the norm of simple functions) or equivalently ( $m \sigma$-finite) if $f$ takes almost all its values in a strongly separable subspace. Thus the function $t \rightarrow \delta_{(t)}$ from $[0,1]$ into $M[0,1]=C[0,1]_{\sigma}^{\prime}$ is totally summable but not Bochner integrable.

If $E=C[0,1]$ concepts $1,2,3$ coincide, but $3,4,5$ are all distinct. If $E=$ $C[0,1]_{\sigma}^{\prime}, 3,4,5$ coincide, but $1,2,3$ are all distinct. Thus if $E=C[0,1] \times$ $C[0,1]_{\sigma}^{\prime}$ all five concepts are distinct.

Grothendieck [8, p. 68], has shown that if $E$ is a separable Fréchet space 1,2, 3 coincide.

We now turn to Fubini's theorem. It is known that Fubini's theorem is valid for Bochner integrable functions (D \& S [6, III.11.9]) while it is not valid for Pettis summable functions even with values in a separable Hilbert space $[1$, p. 376 , Example 6].

(15) This terminology is not established. We adopted it following Pietsch [12, 1.5.1]. 
Let $\left(T_{i}, A_{i}, m_{i}\right), i=1,2$, be two $\sigma$-finite measure spaces. The product measure space is $(T, A, m)$ where $T=T_{1} \times T_{2}, A=A_{1} \otimes A_{2}$ is the smallest $\sigma$-algebra containing the sets $A_{1} \times A_{2}, A_{i} \in \mathrm{A}_{i}$, and $m$ is the product measure.

THEOREM 8. Let $\left(T_{i}, A_{i}, m_{i}\right)$ be two o-finite measure spaces and let $(T, A, m)$ be the product measure space, or, if the given measure spaces are complete, the completion of the product measure space. Let $f: T \rightarrow E$ be totally m-summable. Then we have the following conclusion:

(a) $t_{2} \rightarrow f\left(t_{1}, t_{2}\right)$ is totally $m_{2}$-summable for almost all $t_{1}$ (all except those in a set $N_{1} \in A_{1}$ with $m_{1}\left(N_{1}\right)=0$ ).

(b) $t_{1} \rightarrow \int f\left(t_{1}, t_{2}\right) d m_{2}\left(t_{2}\right)$ is totally $m_{1}$-summable (over $\left.T_{1}-N_{1}\right)$.

(c) $\iint f d m=\int d m_{1}\left(t_{1}\right) \int f\left(t_{1}, t_{2}\right) d m_{2}\left(t_{2}\right)$.

Proof. We first assume $(T, A, m)$ is the product measure space. By hypothesis $\iint|f|_{B} d m<+\infty$ for some $B$. The function $f$ is measurable and so for each $t_{1} \in T_{1}$, and $x^{\prime} \in E^{\prime}, t_{2} \rightarrow\left\langle f\left(t_{1}, t_{2}\right), x^{\prime}\right\rangle$ is measurable whence $f\left(t_{1}, \cdot\right)$ is measurable for all $t_{1}$. Now by Tonelli's theorem $\iint|f|_{B} d m=\int d t_{1} \int\left|f\left(t_{1}, t_{2}\right)\right|_{B} d t_{2}<+\infty$, the function $t_{1} \rightarrow \int\left|f\left(t_{1}, t_{2}\right)\right|_{B} d t_{2}$ being measurable. And so for almost all $t_{1}$ (all except those in a set $N_{1} \in A_{1}$ with $\left.m_{1}\left(N_{1}\right)=0\right) \int\left|f\left(t_{1}, t_{2}\right)\right|_{B} d t_{2}<+\infty$ whence (a). By Fubini's theorem for scalar functions

$$
t_{1} \rightarrow\left\langle\int f\left(t_{1}, t_{2}\right) d t_{2}, x^{\prime}\right\rangle=\int\left\langle f\left(t_{1}, t_{2}\right), x^{\prime}\right\rangle d t_{2}
$$

is measurable on $T_{1}-N_{1}$ and so $t_{1} \rightarrow \int f\left(t_{1}, t_{2}\right) d t_{2}$ is measurable on $T_{1}-N_{1}$. Again by Tonelli's theorem and the inequality

IX $\quad \int_{T_{1}-N_{1}} d t_{1}\left|\int f\left(t_{1}, t_{2}\right) d t_{2}\right|_{B} \leqslant \int d t_{1} \int\left|f\left(t_{1}, t_{2}\right)\right|_{B} d t_{2}<+\infty$,

and this proves (b). Finally

$$
\begin{aligned}
\left\langle\int_{T_{1}-N_{1}} d t_{1} \int f\left(t_{1}, t_{2}\right) d t_{2}, x^{\prime}\right\rangle & =\int_{T_{1}-N_{1}} d t_{1} \int\left\langle f\left(t_{1}, t_{2}\right), x^{\prime}\right\rangle d t_{2} \\
\cdot & =\iint\left\langle f, x^{\prime}\right\rangle d m=\left\langle\iint f d m, x^{\prime}\right\rangle
\end{aligned}
$$

by Fubini's theorem and (c) results.

The proof in the second case is entirely similar if one uses the following lemma: Let $f: T \rightarrow E$ be A-measurable. Then the function $t_{2} \rightarrow f\left(t_{1}, t_{2}\right)$ is $A_{2}$-measurable for almost all $t_{1}$.

Proof. Let $H \subset E^{\prime}$ be a countable total subset. Then by the corresponding result for scalar functions $t_{2} \rightarrow\left\langle f\left(t_{1}, t_{2}\right), x^{\prime}\right\rangle$ is $A_{2}$-measurable for almost all $t_{1}$ and all $x^{\prime} \in H$. For those elements $t_{1}, f\left(t_{1}, \cdot\right)$ is $A_{2}$-measurable by Theorem 1 . 
6. Integration of measures. Given a summable function $f: T \rightarrow E$, the relations $\left\langle\int f d m, x^{\prime}\right\rangle=\int\left\langle f, x^{\prime}\right\rangle d m$ will sometimes hold for linear forms $x^{\prime}$ which are not necessarily continuous on $E$. The most important particular case is expressed in the following:

THEOREM 9. Let $S$ be a locally compact space with a countable base of open sets. Let $M=C_{0}(S)_{\sigma}^{\prime}$ be the space of bounded complex measures with the weak dual topology $\left(\mathrm{C}_{0}(S)\right.$ being the space of continuous functions vanishing at infinity). Then we have:

(a) $t \rightarrow \mu_{t} \in M$ is $m$-summable if and only if $t \rightarrow\left\langle\mu_{t}, \varphi\right\rangle$ is integrable for each $\varphi \in C_{0}$.

(b) If $\mu=\int \mu_{t} d m(t)$ we have $\langle\mu, h\rangle=\int\left\langle\mu_{t}, h\right\rangle d m(t)$ for all bounded Borel functions $h$.

(c) The integral $\int_{A} \mu_{t} d m(t)$ is countably additive in the norm topology of $M$.

Proof. (a) Follows from 4.1. Alternatively let $p(\varphi)=\int\left\langle\mu_{t}, \varphi\right\rangle \mid d m(t)$. By Fatou's theorem $p$ is a lower semicontinuous seminorm, hence continuous since $C_{0}(S)$ is barrelled. Thus if $\left\langle\mu_{A}, \varphi\right\rangle=\int_{A}\left\langle\mu_{t}, \varphi\right\rangle d m(t)$ we have $\left\langle\mu_{A}, \varphi\right\rangle \mid \leqslant p(\varphi) \leqslant$ $M\|\varphi\|_{\infty}$ and so $\mu_{A} \in C_{0}(S)^{\prime}=M \cdot\left({ }^{16}\right)$

(b) Let $B_{1}$ be the set of all Borel functions on $S$ such that (i) $|h| \leqslant 1$, (ii) $t \rightarrow\left\langle\mu_{t}, h\right\rangle$ is measurable, and (iii) $\int\left\langle\mu_{t}, h\right\rangle \mid d m(t) \leqslant M$. Then $B_{1}$ contains all $\varphi \in C_{0}$ with $|\varphi| \leqslant 1$, and is closed under pointwise convergence of sequences, by the dominated convergence theorem and by Fatou's lemma. Thus $B_{1}$ contains all Baire, hence Borel, functions $h$ with $|h| \leqslant 1$. It remains to prove that $\langle\mu, h\rangle=$ $\int\left\langle\mu_{t}, h\right\rangle d m(t)$ for all $h$. Assume first $\int\left\|\mu_{t}\right\| d m(t)<+\infty$. Then by the dominated convergence theorem the set of $h \in B_{1}$ for which the equality holds is closed under pointwise convergence of sequences; hence it includes all of $B_{1}$. In the general case let $T=\Sigma_{n} T_{n}$ with $\int_{T_{n}}\left\|\mu_{t}\right\| d m(t)<+\infty$, and put $\mu_{n}=$ $\int_{T_{n}} \mu_{t} d m(t)$. Then we have

$$
\sum_{n}\left|\left\langle\mu_{n}, h\right\rangle\right| \leqslant \sum \int_{T_{n}}\left|\left\langle\mu_{t}, h\right\rangle\right| d m(t) \leqslant M\|h\|_{\infty}
$$

Let $\nu(B)=\Sigma_{n} \mu_{n}(B), B$ being a Borel subset of $S$. Then $\nu$ is countably additive [6, III.7.4]; $\langle\nu, h\rangle$ and $\Sigma_{n}\left\langle\mu_{n}, h\right\rangle$ coincide on simple functions, hence by continuity on all bounded Borel functions. Putting $h=\varphi \in C_{0}$ we see that $\nu=\mu$, and the proof of (b) is complete. (c) Let $\left(A_{n}\right)$ be a sequence of disjoint measurable sets and let $\mu_{n}=\int_{A_{n}} \mu_{t} d m(t)$. There exists a measure $\lambda \geqslant 0$ such that all

(16) This argument does not use the separability of $C_{0}$ and is valid for functions with values in the dual of any Frechet space. For the proof of (b) we use the separability assumption. 
the measures $\mu_{n}$, and by (b) all their sums, are absolutely continuous with respect to $\lambda$, hence belong to the space $L^{1}(\lambda)$ identified with a closed subspace of $M_{b}$. The most general continuous linear form on $L^{1}(\lambda)$ is of the form $\rho \rightarrow\langle\rho, h\rangle$ where $h$ is a bounded Borel function. The last assertion now results from Orlicz's theorem.

The proof of the following theorem which generalizes Fubini's theorem is left to the reader.

TheOREM 10. Let $(S, B, \mu)$ and $(T, A, m)$ be $\sigma$-finite positive measure spaces. Let $\left(\mu_{t}\right)_{t \in T}$ be a family of positive measures on $(S, B)$ such that

(a) $t \rightarrow \mu_{t}(B)$ is measurable for all $B \in B$.

(b) $\mu(B)=\int \mu_{t}(B) d m(t) \quad \forall B \in B$.

Then if $f: S \rightarrow E$ is totally $\mu$-summable, $f$ is totally $\mu_{t}$-summable for almost all $t, t \rightarrow \int f d \mu_{t}$ is totally m-summable, and $\int f d \mu=\int d m(t) \int f d \mu_{t}$.

7. Differentiation. In this section $T$ denotes an open subset of $\mathbf{R}^{n}, A$ the set of Lebesgue measurable subsets of $T$ and $m$ Lebesgue measure (also noted $d t$, etc). Let $|\cdot|$ denote a norm in $\mathbf{R}^{n}$ and $v(r)$ the measure of the balls of radius $r$.

THEOREM 11. Let $f: T \rightarrow E$ be totally $m$-summable. Then for almost all $t \in T$,

$$
\lim _{r \rightarrow 0} \frac{1}{v(r)} \int_{|t-s|<r}|f(t)-f(s)|_{p} d s=0
$$

for all continuous seminorms $p$. In particular

$$
f(t)=\lim _{r \rightarrow 0} \frac{1}{v(r)} \int_{|s-t|<r} f(s) d s \text { for almost all } t .
$$

If $E$ is metrisable the theorem follows immediately from Bochner's theorem $[2, \S 5]$ applied to the spaces $E_{p_{n}}$ associated with the seminorms of some fundamental sequence. Actually, since in this case $f$ is Bochner integrable (cf. §5), there exists a bounded set $B$ such that

$$
\lim _{r \rightarrow 0} \frac{1}{v(r)} \int_{|t-s|<r}|f(t)-f(s)|_{B} d s=0 .
$$

But if uncountably many seminorms are involved we cannot apply Bochner's theorem directly. Neither does there exist a set $B$ for which the above relation is valid (even though $\int|f(t)|_{B} d t<+\infty$ ). This is illustrated by the function $t \rightarrow \delta_{(t)}$ from $[0,1]$ to $M[0,1]_{\sigma}$. 
The proof of Theorem 11 will be given elsewhere. It is based on the relation between differentiation and Lusin's theorem (first observed by Denjoy). The case of a totally summable function with values in the weak dual of a separable Banach space can be very easily treated ad hoc.

\section{REFERENCES}

1. G. Birkhoff, Integration of functions with values in a Banach space, Trans. Amer. Math. Soc. 38 (1935), 357-378.

2. S. Bochner, Integration von Funktionen, derer Werte die Elemente eines Vectorräumes sind, Fund. Math 20 (1938), 262-276.

3. N. Bourbaki, Eléments de mathématique. XVIII. Part 1: Les structures fondamentales de l'analyse. Livre V: Espaces vectoriels topologiques. Chap. 4, Actualités Sci. Indust., no. 1229, Hermann, Paris, 1955. MR 17, 1109.

4. - Eléments de mathématique. XXV. Part 1: Les structures fondamentales de l'analyse. Livre VI: Intégration. Chap. 6, Actualités Sci. Indust., no. 1281, Hermann, Paris, 1959. MR 23 \#A2033.

5. J. P. R. Christensen, Borel structures and a topological zero-one law, Math. Scand. 29 (1971), 245-255. MR 47 \#2021.

6. N. Dunford and J. T. Schwartz, Linear operators. I: General theory, Pure and Appl. Math., vol. 7., Interscience, New York, 1958. MR 22 \#8302.

7. L. M. Graves, Riemann integration and Taylor's theorem in general analysis, Trans. Amer. Math. Soc. 29 (1927), 163-177.

8. A. Grothendieck, Produits tensoriels topologiques et espaces nucléaires, Chap. 1 : Produits tensoriels topologiques, Mem. Amer. Math. Soc. No. 16 (1955). MR 17, 763.

9. - Produits tensoriels topologiques et espaces nucléaires, Chap. 2: Espaces nu. cléaires, Mem. Amer. Math. Soc. No. 16 (1955). MR 17, 763.

10. T. H. Hildebrandt, Integration in abstract spaces, Bull. Amer. Math. Soc. 59 (1953), 111-139. MR 14, 735.

11. B. J. Pettis, On integration in vector spaces. Trans. Amer. Math. Soc. 44 (1938), 277-304.

12. A. Pietsch, Nukleare Lokalkonvexe Räume, Akademie-Verlag, Berlin, 1965. MR 31 \#6114.

13. L. Schwartz, Radon measures on arbitrary topological spaces and cylindrical measures, Publ. Tata Inst. of Fundamental Research, Oxford Univ. Press, 1973.

14. G. E. F. Thomas, The Lebesgue-Nikodym theorem for vector valued Radon measures, Mem. Amer. Math. Soc. No. 139 (1974).

\section{DEPARTMENT OF MATHEMATICS, YALE UNIVERSITY, NEW HAVEN, CONNECT. ICUT 06520}

Current address: Mathematisch Instituut, Rijks Universiteit van Groningen, Postbus 800 , Groningen, The Netherlands 